Journal of Mathematics and Statistics 5 (1): 77-87, 2009

ISSN 1549-3644

(C) 2009 Science Publications

\title{
On New Bijective Convolution Operator Acting for Analytic Functions
}

\author{
Oqlah Al-Refai and Maslina Darus \\ School of Mathematical Sciences, Faculty of Science and Technology, \\ Universiti Kebangsaan Malaysia, Bangi 43600, Selangor, Darul Ehsan, Malaysia
}

\begin{abstract}
Problem statement: We introduced a new bijective convolution linear operator defined on the class of normalized analytic functions. This operator was motivated by many researchers namely Srivastava, Owa, Ruscheweyh and many others. The operator was essential to obtain new classes of analytic functions. Approach: Simple technique of Ruscheweyh was used in our preliminary approach to create new bijective convolution linear operator. The preliminary concept of Hadamard products was mentioned and the concept of subordination was given to give sharp proofs for certain sufficient conditions of the linear operator aforementioned. In fact, the subordinating factor sequence was used to derive different types of subordination results. Results: Having the linear operator, subordination theorems were established by using standard concept of subordination. The results reduced to wellknown results studied by various researchers. Coefficient bounds and inclusion properties, growth and closure theorems for some subclasses were also obtained. Conclusion: Therefore, many interesting results could be obtained and some applications could be gathered.
\end{abstract}

Key words: Convolution, convex functions, starlike functions, prestarlike functions, subordinations

\section{INTRODUCTION}

Let $\mathcal{A}$ denote the class of functions $\mathrm{f}$ normalized by:

$f(z)=z+\sum_{n=1}^{\infty} a_{n} z^{n+1}$

which are analytic in the open unit disk $\mathbb{U}=\{\mathrm{z}:|\mathrm{z}|<1\}$, let $\mathcal{T}$ denote the subclass of $\mathcal{A}$ consisting of functions of the form:

$$
f(z)=z-\sum_{n=1}^{\infty} a_{n} z^{n+1},\left(a_{n} \geq 0, z \in \mathbb{U}\right)
$$

We denote also by $\mathrm{K}$ the class of functions $\mathrm{f} \in \mathcal{A}$ that are convex in $\mathbb{U}$ :

Given two functions $\mathrm{f}, \mathrm{g} \in \mathcal{A}$, where

$$
\mathrm{f}(\mathrm{z})=\sum_{\mathrm{n}=0}^{\infty} \mathrm{a}_{\mathrm{n}} \mathrm{z}^{\mathrm{n}+1} \text { and } \mathrm{g}(\mathrm{z})=\sum_{\mathrm{n}=0}^{\infty} \mathrm{b}_{\mathrm{n}} \mathrm{z}^{\mathrm{n}+1}
$$

their Hadamard product or convolution $\mathrm{f} * \mathrm{~g}$ is defined by:

$\mathrm{f}(\mathrm{z}) * \mathrm{~g}(\mathrm{z})=\sum_{\mathrm{n}=0}^{\infty} \mathrm{a}_{\mathrm{n}} \mathrm{b}_{\mathrm{n}} \mathrm{z}^{\mathrm{n}+1} \quad(\mathrm{z} \in \mathbb{U})$
Using the convolution techniques, Ruscheweyh ${ }^{[1]}$ introduced and studied the class of prestarlike functions of order $\beta$. Thus $\mathrm{f} \in \mathcal{A}$ is said to be prestarlike function of order $\beta(0 \leq \beta<1)$ if $\mathrm{f}(\mathrm{z}) * \mathrm{~s}_{\beta}(\mathrm{z})$ is starlike function of order $\beta$, where:

$$
\mathrm{s}_{\beta}(\mathrm{z})=\frac{\mathrm{z}}{(1-\mathrm{z})^{2(1-\beta)}}=\mathrm{z}+\sum_{\mathrm{n}=1}^{\infty} \mathrm{C}(\beta, \mathrm{n}+1) \mathrm{z}^{\mathrm{n}+1}
$$

and

$$
\mathrm{C}(\beta, \mathrm{n}+1)=\frac{\prod_{\mathrm{j}=1}^{\mathrm{n}+1}(\mathrm{j}+1-2 \beta)}{\mathrm{n} !}
$$

Let the function $\varphi(a, b ; z)$ be given by:

$$
\varphi(a, b ; z):=\sum_{n=0}^{\infty} \frac{(a)_{n}}{(b)_{n}} z^{n+1} \quad(b \neq 0,-1,-2, \ldots . ; z \in \mathbb{U})
$$

where $(\mathrm{x})_{\mathrm{n}}$ is the Pochhammer symbol defined by:

$$
(\mathrm{x})_{\mathrm{n}}:=\frac{\Gamma(\mathrm{x}+\mathrm{n})}{\Gamma(\mathrm{x})}=\left\{\begin{array}{cc}
1, & \mathrm{n}=0 \\
\mathrm{x}(\mathrm{x}+1)(\mathrm{x}+2) \ldots(\mathrm{x}+\mathrm{n}-1), & \mathrm{n} \in \mathbb{N}:=\{1,2,3, \ldots\}
\end{array}\right.
$$

Corresponding Auhtor: Maslina Darus, School of Mathematical Sciences, Faculty of Science and Technology, University Kebangsaan Malaysia, Bangi 43600, Selangor, Darul Ehsan, Malaysia 
Note that $\quad(x+1)_{n}=(x+n)(x)_{n} / x$,
$(x-1)(x)_{n-1}=(x-1)_{n}$ and $\frac{\mathrm{z}}{(1-z)^{\beta}}=\sum_{n=0}^{\infty} \frac{(\beta)_{n}}{n !} z^{n+1} \quad(\beta>0)$.

The function $\varphi(a, b ; z)$ is an incomplete beta function related to the Gauss hypergeometric function by $\varphi(\mathrm{a}, \mathrm{b} ; \mathrm{z})=\mathrm{z}_{2} \mathrm{~F}_{1}(1, \mathrm{a}, \mathrm{b}, \mathrm{z})$, where the hypergeometric function ${ }_{2} \mathrm{~F}_{1}(\mathrm{a}, \mathrm{b}, \mathrm{c}, \mathrm{z})$ is defined by:

$$
{ }_{2} \mathrm{~F}_{1}(\mathrm{a}, \mathrm{b}, \mathrm{c}, \mathrm{z})=1+\frac{\mathrm{ab}}{\mathrm{c}} \frac{\mathrm{z}}{1 !}+\frac{\mathrm{a}(\mathrm{a}+1) \mathrm{b}(\mathrm{b}+1)}{\mathrm{c}(\mathrm{c}+1)} \frac{\mathrm{z}^{2}}{2 !}+\ldots
$$

Also, it has an analytic continuation to the z-plane cut along the positive real line from 1 to $\infty$. We note that $\varphi(a, 1 ; z)=z /(1-z)^{a}$ and $\varphi(2,1 ; z)$ is the Koebe function.

Corresponding to the function $\varphi(\mathrm{a}, \mathrm{b} ; \mathrm{z})$, Carlson and Shaffer introduced in $^{[2]}$ a convolution operator on $\mathcal{A}$ involving an incomplete beta function as:

$L(a, b) f(z):=\varphi(a, b ; z) * f(z)=\sum_{n=0}^{\infty} \frac{(a)_{n}}{(b)_{n}} a_{n} z^{n+1}$

The Ruscheweyh derivatives of $\mathrm{f}(\mathrm{z})$ are $\mathrm{L}(\beta+1,1) \mathrm{f}(\mathrm{z}),(\beta \geq-1)$.

\section{MATERIALS AND METHODS}

Using the Hadamard product (or convolution), we define a new bijective convolution operator acts on analytic functions in $\mathbb{U}$. Then we state some of its properties and its special cases which will be used in this study. Corresponding to our operator, we define classes of analytic functions look like the classes of starlike and convex functions of order $\alpha(0 \leq \alpha<1)$. Subordination principle and known results of subordination factor sequence will be used in our investigation of that classes.

Definition 1: Let the function $\phi$ be given by:

$$
\phi(m, \lambda, a, b ; z):=\sum_{n=0}^{\infty}(1+\lambda n)^{m} \frac{(a)_{n}}{(b)_{n}} z^{n+1}
$$

where, $(b \neq 0,-1,-2, \ldots . ; z \in \mathbb{U}), \lambda \geq 0, m \in \mathbb{Z}$ and $(x)_{n}$ is the Pochhammer symbol. One defines a linear operator $\mathrm{J}(\mathrm{m}, \lambda, \mathrm{a}, \mathrm{b}): \mathcal{A} \rightarrow \mathcal{A}$ by the following Hadamard product (or convolution):

$$
\begin{aligned}
\mathrm{J}(\mathrm{m}, \lambda, \mathrm{a}, \mathrm{b}) \mathrm{f}(\mathrm{z}) & :=\phi(\mathrm{m}, \lambda, \mathrm{a}, \mathrm{b} ; \mathrm{z}) * \mathrm{f}(\mathrm{z}) \\
& =\sum_{\mathrm{n}=0}^{\infty}(1+\lambda \mathrm{n})^{\mathrm{m}} \frac{(\mathrm{a})_{\mathrm{n}}}{(\mathrm{b})_{\mathrm{n}}} \mathrm{a}_{\mathrm{n}} \mathrm{z}^{\mathrm{n}+1}
\end{aligned}
$$

Note that:

$$
\begin{aligned}
\mathrm{J}(0, \lambda, \mathrm{a}, \mathrm{b}) \mathrm{f}(\mathrm{z}) & =\mathrm{L}(\mathrm{a}, \mathrm{b}) \mathrm{f}(\mathrm{z}) \\
\mathrm{J}(1, \lambda, \mathrm{a}, \mathrm{b}) \mathrm{f}(\mathrm{z}) & =(1-\lambda) \mathrm{L}(\mathrm{a}, \mathrm{b}) \mathrm{f}(\mathrm{z})+\lambda \mathrm{z}(\mathrm{L}(\mathrm{a}, \mathrm{b}) \mathrm{f}(\mathrm{z}))^{\prime} \\
& =\mathrm{J}_{\lambda}(\mathrm{L}(\mathrm{a}, \mathrm{b}) \mathrm{f}(\mathrm{z})), \lambda \geq 0 \\
\mathrm{~J}(\mathrm{~m}, \lambda, \mathrm{a}, \mathrm{b}) \mathrm{f}(\mathrm{z}) & =\mathrm{J}_{\lambda}(\mathrm{J}(\mathrm{m}-1, \lambda, \mathrm{a}, \mathrm{b}) \mathrm{f}(\mathrm{z})), \text { where } \\
\mathrm{m} \in \mathbb{N} & =\{1,2,3, \ldots\}
\end{aligned}
$$

If $\mathrm{a}=0,-1,-2, \ldots$, then $\mathrm{J}(\mathrm{m}, \lambda, \mathrm{a}, \mathrm{b}) \mathrm{f}(\mathrm{z})$ is $\mathrm{a}$ polynomial. If $a \neq 0,-1,-2$, then application of the root test shows that the infinite series for $\mathrm{J}(\mathrm{m}, \lambda, \mathrm{a}, \mathrm{b}) \mathrm{f}(\mathrm{z})$ has the same radius of convergence as that for $f$ because:

$$
\begin{aligned}
& \lim _{n \rightarrow \infty}\left|[1+\lambda n]^{m} \frac{(a)_{n}}{(b)_{n}}\right|=\lim _{n \rightarrow \infty}\left|\left[(1+\lambda n) \frac{(a)_{n}}{(b)_{n}}\right]^{m}\left[\frac{(a)_{n}}{(b)_{n}}\right]^{1-m}\right| \\
& =\lim _{n \rightarrow \infty}\left|\begin{array}{l}
\left.\lambda a \frac{(a+1)_{n}}{(b)_{n}}-(\lambda a-1) \frac{(a)_{n}}{(b)_{n}}\right]^{m} \\
{\left[\frac{(a)_{n}}{(b)_{n}}\right]^{1-m}}
\end{array}\right| \\
& =\left|\begin{array}{l}
\left.\lambda a \lim _{n \rightarrow \infty} \frac{(a+1)_{n}}{(b)_{n}}-(\lambda a-1) \lim _{n \rightarrow \infty} \frac{(a)_{n}}{(b)_{n}}\right]^{m} \\
{\left[\lim _{n \rightarrow \infty} \frac{(a)_{n}}{(b)_{n}}\right]^{1-m}}
\end{array}\right|
\end{aligned}
$$

and the last expression equal 1 since:

$$
\lim _{n \rightarrow \infty}\left|\frac{(a)_{n}}{(b)_{n}}\right|=1
$$

Hence, $\mathrm{J}(\mathrm{m}, \lambda, \mathrm{a}, \mathrm{b})$ maps $\mathcal{A}$ into itself. So, we shall assume, unless other wise stated, that $(a \neq 0,-1,-2, \ldots)$ and $(b \neq 0,-1,-2, \ldots)$. We denote by $\mathrm{g}^{-1}$ where $\mathrm{g} \in \mathcal{A}$, the function which satisfies $\mathrm{g}(\mathrm{z}) * \mathrm{~g}^{-1}(\mathrm{z})=\mathrm{z} / 1-\mathrm{z} ;(\mathrm{z} \in \mathbb{U})$. For $\mathrm{a}>0$ and $\mathrm{b}>0$, if $\lambda=0$, then:

$$
\mathrm{J}(\mathrm{m}, 0, \mathrm{a}, \mathrm{b}) \mathrm{f}(\mathrm{z})=\mathrm{L}(\mathrm{a}, \mathrm{b}) \mathrm{f}(\mathrm{z})=\frac{\mathrm{z}}{(1-\mathrm{z})^{\mathrm{a}}} * \mathrm{z}_{2} \mathrm{~F}_{1}(1,1, \mathrm{~b}, \mathrm{z}) * \mathrm{f}(\mathrm{z}) .
$$

If $\lambda>0$, then the operator $\mathrm{J}(\mathrm{m}, \lambda, \mathrm{a}, \mathrm{b}) \mathrm{f}(\mathrm{z})$ can be represented by:

$$
\begin{aligned}
\mathrm{J}(\mathrm{m}, \lambda, \mathrm{a}, \mathrm{b}) \mathrm{f}(\mathrm{z})= & \frac{\mathrm{z}}{(1-\mathrm{z})^{\mathrm{a}}} * \mathrm{z}_{2} \mathrm{~F}_{1}(1,1, \mathrm{~b}, \mathrm{z}) * \frac{\mathrm{z}}{\underbrace{1+\frac{1}{\lambda}}_{\mathrm{m}} * \ldots * \frac{\mathrm{z}}{(1-\mathrm{z})^{1+\frac{1}{\lambda}}}} \\
& * \underbrace{\mathrm{z}_{2} \mathrm{~F}_{1}(1,1,1 / \lambda, \mathrm{z}) * \ldots * \mathrm{z}_{2} \mathrm{~F}_{1}(1,1,1 / \lambda, \mathrm{z})}_{\mathrm{m}-\mathrm{times}} * \mathrm{f}(\mathrm{z})
\end{aligned}
$$


if $(\mathrm{m}=0,1,2, \ldots)$ and

$$
\begin{aligned}
& J(m, \lambda, a, b) f(z)=\frac{z}{(1-z)^{a}} * z_{2} F_{1}(1,1, b, z) * \frac{z}{\frac{(1-z)^{\frac{1}{\lambda}}}{(-m)-t . t i m e s}} \\
& \underbrace{* \mathrm{Z}_{2} \mathrm{~F}_{1}(1,1,1+1 / \lambda, \mathrm{z}) * \ldots * * \mathrm{Z}_{2} \mathrm{~F}_{1}(1,1,1+1 / \lambda, \mathrm{z})} * \mathrm{f}(\mathrm{z})
\end{aligned}
$$

if $(m=-1,-2, \ldots)$.

Note that $\mathrm{J}(0, \lambda, a, a)=J(m, 0, a, a)$ is the identity operator and if $\mathrm{a} \neq 0,-1,-2, \ldots$, then $\mathrm{J}(\mathrm{m}, \lambda, \mathrm{a}, \mathrm{b})$ has a continuous inverse $\mathrm{J}(-\mathrm{m}, \lambda, \mathrm{b}, \mathrm{a})$ and is a one-to-one mapping of $\mathcal{A}$ onto itself. Hence, $\mathrm{J}(\mathrm{m}, \lambda, \mathrm{a}, \mathrm{b})$ maps $\mathcal{A}$ onto itself, infact it maps the class of analytic functions in $\mathbb{U}$ into itself. It also provides a convenient representation of differentiation and integration. By specializing the parameters $\mathrm{m}, \lambda$, a and $\mathrm{b}$, one can obtain various operators, which are special cases of $\mathrm{J}(\mathrm{m}, \lambda, \mathrm{a}, \mathrm{b})$ studied earlier by many authors, such of those operators:

- $\quad J(m, 0, a, b) f(z)=J(0, \lambda, a, b) f(z)=L(a, b) f(z)$

- The Ruscheweyh derivatives $\mathrm{J}(0,0, \beta+1,1) \mathrm{f}(\mathrm{z})=\mathrm{D}^{\beta} \mathrm{f}(\mathrm{z}) ; \quad \beta \geq-1^{[3]}$

- $\mathrm{J}(\mathrm{m}, \lambda, 1,1) \mathrm{f}(\mathrm{z}) ; \mathrm{m} \in \mathbb{N}$ due to Al-Oboudi ${ }^{[4]}$

- $J(m, 1,1,1) f(z) ; m \in \mathbb{N}$ due to $S a ̆ l a ̆$ gean ${ }^{[5]}$

- The fractional operator due to Owa and Srivastava

$\mathrm{J}(0,0,2,2-\gamma) \mathrm{f}(\mathrm{z})=\Omega^{\gamma} \mathrm{f}(\mathrm{z})=\Gamma(2-\gamma) \mathrm{z}^{\gamma} \mathrm{D}_{\mathrm{z}}^{\gamma} \mathrm{f}(\mathrm{z}) ; \quad \mathrm{D}_{\mathrm{z}}^{\gamma} \mathrm{f}(\mathrm{z})$

is the fractional derivative of $\mathrm{f}$ of order $\gamma$; $\gamma \neq 2,3,4, . .,{ }^{[6]}$.

Also, note that the operators $\mathrm{J}(0,0,2, \mathrm{n}+1) \mathrm{f}(\mathrm{z})$; $\mathrm{n} \in \mathbb{N}$ due to $\left.\operatorname{Noor}^{[7,8]}\right)$ and $\mathrm{J}(0,0, \mathrm{~b}, \mathrm{a}) \mathrm{f}(\mathrm{z}) ; \mathrm{a}>0, \mathrm{~b}>0$ due to Choi al et. ${ }^{[9]}$ are special cases of the inverse operator $\mathrm{J}(-\mathrm{m}, \lambda, \mathrm{b}, \mathrm{a}) \mathrm{f}(\mathrm{z})$.

We prove now the following two identities which will be used in this study.

Lemma 1: Let $\mathrm{f} \in \mathcal{A}$ satisfies (4). Then we have the following:

- $\mathrm{z}(\mathrm{J}(\mathrm{m}, \lambda, \mathrm{a}, \mathrm{b}) \mathrm{f}(\mathrm{z}))^{\prime}=\mathrm{aJ}(\mathrm{m}, \lambda, \mathrm{a}+1, \mathrm{~b}) \mathrm{f}(\mathrm{z})-(\mathrm{a}-1) \mathrm{J}(\mathrm{m}$, $\lambda, a, b) f(z)$

- For $(b \neq 1,0,-1, \ldots) z(J(m, \lambda, a, b) f(z))^{\prime}=(b-1) J(m$, $\lambda, a, b-1) f(z)-(b-2) J(m, \lambda, a, b) f(z)$

Proof: Let $J(m, \lambda, a, b) f(z)$ be as in (4). Then we have:

$$
\begin{aligned}
\mathrm{aJ}(\mathrm{m}, \lambda, \mathrm{a}+1, \mathrm{~b}) \mathrm{f}(\mathrm{z})= & \mathrm{a} \sum_{\mathrm{n}}^{\infty}(1+\lambda \mathrm{n})^{\mathrm{m}}\left(1+\frac{\mathrm{n}}{\mathrm{a}}\right) \frac{(\mathrm{a})_{\mathrm{n}}}{(\mathrm{b})_{\mathrm{n}}} \mathrm{a}_{\mathrm{n}} \mathrm{z}^{\mathrm{n}+1} \\
= & \mathrm{aJ}(\mathrm{m}, \lambda, \mathrm{a}, \mathrm{b}) \mathrm{f}(\mathrm{z}) \\
& +\sum_{\mathrm{n}=0}^{\infty} \mathrm{n}(1+\lambda \mathrm{n})^{\mathrm{m}} \frac{(\mathrm{a})_{\mathrm{n}}}{(\mathrm{b})_{\mathrm{n}}} \mathrm{a}_{\mathrm{n}} \mathrm{z}^{\mathrm{n}+1} \\
= & (\mathrm{a}-1) \mathrm{J}(\mathrm{m}, \lambda, \mathrm{a}, \mathrm{b}) \mathrm{f}(\mathrm{z}) \\
+ & \sum_{\mathrm{n}=0}^{\infty}(\mathrm{n}+1)(1+\lambda \mathrm{n})^{\mathrm{m}} \frac{(\mathrm{a})_{\mathrm{n}}}{(\mathrm{b})_{\mathrm{n}}} \mathrm{a}_{\mathrm{n}} \mathrm{z}^{\mathrm{n}+1} \\
(\mathrm{~b}-1) \mathrm{J}(\mathrm{m}, \lambda, \mathrm{a}, \mathrm{b}-1) \mathrm{f}(\mathrm{z})= & \sum_{\mathrm{n}=0}^{\infty}(1+\lambda \mathrm{n})^{\mathrm{m}}(\mathrm{b}+\mathrm{n}-1) \\
& \frac{(\mathrm{a})_{\mathrm{n}}}{(\mathrm{b})_{\mathrm{n}}} \mathrm{a}_{\mathrm{n}} \mathrm{z}^{\mathrm{n}+1} \\
= & (\mathrm{b}-2) \mathrm{J}(\mathrm{m}, \lambda, \mathrm{a}, \mathrm{b}) \mathrm{f}(\mathrm{z}) \\
& +\sum_{\mathrm{n}=0}^{\infty}(\mathrm{n}+1)(1+\lambda \mathrm{n})^{\mathrm{m}} \\
& \frac{(\mathrm{a})_{\mathrm{n}}}{(b)_{\mathrm{n}}} \mathrm{a}_{\mathrm{n}} \mathrm{z}^{\mathrm{n}+1}
\end{aligned}
$$

where $(b \neq 1,0,-1,-2, \ldots)$. Hence, the proof is complete.

Now, we introduce new classes of analytic functions involving our operator $\mathrm{J}(\mathrm{m}, \lambda, \mathrm{a}, \mathrm{b})$.

Definition 2: A function $\mathrm{f} \in \mathcal{A}$ is said to be in the class $\mathrm{S}(\mathrm{m}, \lambda, \mathrm{a}, \mathrm{b}, \alpha)$ for $\mathrm{m} \in \mathbb{Z}, \lambda \geq 0,0 \leq \alpha<1$, if and only if:

$\Re\left\{\frac{\mathrm{z}(\mathrm{J}(\mathrm{m}, \lambda, \mathrm{a}, \mathrm{b}) \mathrm{f}(\mathrm{z}))^{\prime}}{\mathrm{J}(\mathrm{m}, \lambda, \mathrm{a}, \mathrm{b}) \mathrm{f}(\mathrm{z})}\right\}>\alpha$

for all $\mathrm{z} \in \mathbb{U}$

Definition 3: A function $\mathrm{f} \in \mathcal{A}$ is said to be in the class $\mathrm{C}(\mathrm{m}, \lambda, \mathrm{a}, \mathrm{b}, \alpha)$ for $\mathrm{m} \in \mathbb{Z}, \lambda \geq 0,0 \leq \alpha<1$, if and only if:

$\Re\left\{\frac{\left(\mathrm{z}(\mathrm{J}(\mathrm{m}, \lambda, \mathrm{a}, \mathrm{b}) \mathrm{f}(\mathrm{z}))^{\prime}\right)^{\prime}}{(\mathrm{J}(\mathrm{m}, \lambda, \mathrm{a}, \mathrm{b}) \mathrm{f}(\mathrm{z}))^{\prime}}\right\}>\alpha$

for all $z \in \mathbb{U}$.

Remark 1: $f \in S(0,0,2(1-\alpha), 1, \alpha)$ if and only if $f$ is prestarlike function of order $\alpha$.

Definition $4^{[10]}$ : Let $g$ be analytic and univalent in $\mathbb{U}$. If $f$ is analytic in $\mathbb{U}, f(0)=g(0)$ and $f(\mathbb{U}) \subset g(\mathbb{U})$, then one says that $\mathrm{f}$ is subordinate to $\mathrm{g}$ in $\mathbb{U}$ and we write $\mathrm{f} \prec \mathrm{g}$ or $\mathrm{f}(\mathrm{z}) \prec \mathrm{g}(\mathrm{z})$. One also says that $\mathrm{g}$ is superordinate to $f$ in $\mathbb{U}$. 
Definition $5^{[10]}$ : An infinite sequence $\left\{b_{k}\right\}_{k=1}^{\infty}$ of complex numbers is said to be a subordinating factor sequence if for every univalent function $\mathrm{f}$ in $\mathrm{K}$, one has:

$$
\sum_{\mathrm{k}=1}^{\infty} \mathrm{b}_{\mathrm{k}} \mathrm{a}_{\mathrm{k}} \mathrm{z}^{\mathrm{k}} \prec \mathrm{f}(\mathrm{z}) \quad\left(\mathrm{z} \in \mathbb{U} ; \mathrm{a}_{1}=1\right)
$$

Lemma $2^{[11]}$ : The sequence $\left\{b_{k}\right\}_{k=1}^{\infty}$ is a subordinating factor sequence if and only if:

$$
\Re\left\{1+2 \sum_{\mathrm{k}=1}^{\infty} \mathrm{b}_{\mathrm{k}} \mathrm{z}^{\mathrm{k}}\right\}>0 \quad(\mathrm{z} \in \mathbb{U})
$$

\section{RESULTS}

Coefficient bounds and Inclusions: we investigate sufficient conditions for the function $\mathrm{f} \in \mathcal{A}$ to be in the classes $\mathrm{S}(\mathrm{m}, \lambda, \mathrm{a}, \mathrm{b}, \alpha)$ and $\mathrm{C}(\mathrm{m}, \lambda, \mathrm{a}, \mathrm{b}, \alpha)$ by obtaining the coefficient bounds. Moreover, we study some inclusion properties for some subclasses of $\mathrm{S}(\mathrm{m}, \lambda, \mathrm{a}, \mathrm{b}, \alpha)$ and $\mathrm{C}(\mathrm{m}, \lambda, \mathrm{a}, \mathrm{b}, \alpha)$.

Theorem 1: Let $\mathrm{f} \in \mathcal{A}$. If

$$
\sum_{n=1}^{\infty}(n+1-\alpha)(1+\lambda n)^{m}\left|\frac{(a)_{n}}{(b)_{n}}\right| a_{n} \mid \leq 1-\alpha
$$

then $f \in S(m, \lambda, a, b, \alpha)$, where $S(m, \lambda, a, b, \alpha)$ is defined as in Definition 2.

Proof: Suppose that (7) holds. Then by using Lemma 1 and for all $\mathrm{z} \in \mathbb{U}$, we have:

$$
\begin{aligned}
& \left|\frac{\mathrm{z}(\mathrm{J}(\mathrm{m}, \lambda, \mathrm{a}, \mathrm{b}) \mathrm{f}(\mathrm{z}))^{\prime}}{\mathrm{J}(\mathrm{m}, \lambda, \mathrm{a}, \mathrm{b}) \mathrm{f}(\mathrm{z})}-1\right|=|\mathrm{a}| \frac{\mathrm{J}(\mathrm{m}, \lambda, \mathrm{a}+1, \mathrm{~b}) \mathrm{f}(\mathrm{z})}{\mathrm{J}(\mathrm{m}, \lambda, \mathrm{a}, \mathrm{b}) \mathrm{f}(\mathrm{z})}-1 \mid \\
& =|a| \frac{\sum_{n=0}^{\infty}(1+\lambda n)^{m} \frac{(a+1)_{n}-(a)_{n}}{(b)_{n}} a_{n} z^{n+1}}{\sum_{n=0}^{\infty}(1+\lambda n)^{m} \frac{(a)_{n}}{(b)_{n}} a_{n} z^{n+1}} \mid \\
& \leq \frac{\sum_{n=1}^{\infty}(1+\lambda n)^{m}|a| \frac{(a+1)_{n}-(a)_{n}}{(b)_{n}}\left|a_{n}\right|}{1-\sum_{n=1}^{\infty}(1+\lambda n)^{m}\left|\frac{(a)_{n}}{(b)_{n}}\right| a_{n} \mid}
\end{aligned}
$$

The last expression is bounded by $(1-\alpha)$ if the following inequality which is equivalent to (7) holds:

$$
\sum_{n=1}^{\infty}(1+\lambda n)^{m}\left|\frac{a(a+1)_{n}-(a+\alpha-1)(a)_{n}}{(b)_{n}}\right| a_{n} \mid \leq 1-\alpha
$$

and this implies $f \in S(m, \lambda, a, b, \alpha)$. This completes the proof of Theorem 1.

Let $\mathrm{S}^{*}(\mathrm{~m}, \lambda, \mathrm{a}, \mathrm{b}, \alpha)$ denote the class of functions $\mathrm{f} \in \mathcal{A}$ whose Taylor-Maclaurin coefficients $a_{\mathrm{n}}$ satisfy the condition (7) and denote $\mathrm{S}^{*}[\mathrm{~m}, \lambda, a, b, \alpha]=\mathrm{S}^{*}(\mathrm{~m}, \lambda, \mathrm{a}, \mathrm{b}, \alpha) \cap \mathcal{T}$. We note that:

$$
\mathrm{S}^{*}(\mathrm{~m}, \lambda, \mathrm{a}, \mathrm{b}, \alpha) \subseteq \mathrm{S}(\mathrm{m}, \lambda, \mathrm{a}, \mathrm{b}, \alpha) .
$$

Corollary 1: Let $\mathrm{f} \in \mathcal{A}$ be in the class $\mathrm{S}^{*}(\mathrm{~m}, \lambda, \mathrm{a}, \mathrm{b}, \alpha)$. Then:

$$
\left|a_{n}\right| \leq \frac{1-\alpha}{(n+1-\alpha)(1+\lambda n)^{m}}\left|\frac{(b)_{n}}{(a)_{n}}\right|
$$

Example 1: The function $f$ given by:

$$
f(z)=z \pm \sum_{n=1}^{\infty} \frac{(1-\alpha) \alpha^{n} \varepsilon_{n}(b)_{n}}{(n+1-\alpha)(1+\lambda n)^{m}(a)_{n}} z^{n+1}
$$

belongs to the class $\mathrm{S}^{*}(\mathrm{~m}, \lambda, \mathrm{a}, \mathrm{b}, \alpha)$ for $0 \leq \alpha \leq \frac{1}{2}$, $\mathrm{m} \in \mathbb{Z}, \lambda \geq 0$ and $\varepsilon_{\mathrm{n}} \in \mathbb{C}$ with $\left|\varepsilon_{\mathrm{n}}\right|=1$.

Example 2: For $0 \leq \alpha<1, \lambda \geq 0$ and $m \in \mathbb{N}_{0}$, the following functions defined by:

$$
f_{n}(z)=z \pm \frac{(1-\alpha)}{(n+1-\alpha)(1+\lambda n)^{m}}\left|\frac{(b)_{n}}{(a)_{n}}\right| z^{n+1} \quad(n=1,2, \ldots ; z \in \mathbb{U})
$$

are in the class $\mathrm{S}^{*}(\mathrm{~m}, \lambda, \mathrm{a}, \mathrm{b}, \alpha)$.

Theorem 2: Let $\mathrm{f} \in \mathcal{A}$. If:

$\sum_{n=1}^{\infty}(n+1)(n+1-\alpha)(1+\lambda n)^{m}\left|\frac{(a)_{n}}{(b)_{n}}\right|\left|a_{n}\right| \leq 1-\alpha$

then $f \in C(m, \lambda, a, b, \alpha)$, where $C(m, \lambda, a, b, \alpha)$ is defined as in Definition 3.

Proof: Suppose that (8) holds. Then by using Lemma 1 (i), with differentiating its both sides and for all $z \in \mathbb{U}$, we have: 


$$
\begin{aligned}
\left|\frac{\mathrm{z}(\mathrm{J}(\mathrm{m}, \lambda, \mathrm{a}, \mathrm{b}) \mathrm{f}(\mathrm{z}))^{\prime \prime}}{(\mathrm{J}(\mathrm{m}, \lambda, \mathrm{a}, \mathrm{b}) \mathrm{f}(\mathrm{z}))^{\prime}}\right| & =|\mathrm{a}|\left|\frac{(\mathrm{a}+1) \mathrm{J}(\mathrm{m}, \lambda, \mathrm{a}+2, \mathrm{~b}) \mathrm{f}(\mathrm{z})-2 \mathrm{aJ}(\mathrm{m}, \lambda, \mathrm{a}+1, \mathrm{~b}) \mathrm{f}(\mathrm{z})+(\mathrm{a}-1) \mathrm{J}(\mathrm{m}, \lambda, \mathrm{a}, \mathrm{b}) \mathrm{f}(\mathrm{z})}{\mathrm{aJ}(\mathrm{m}, \lambda, \mathrm{a}+1, \mathrm{~b}) \mathrm{f}(\mathrm{z})-(\mathrm{a}-1) \mathrm{J}(\mathrm{m}, \lambda, \mathrm{a}, \mathrm{b}) \mathrm{f}(\mathrm{z})}\right| \\
& \leq \frac{\sum_{\mathrm{n}=1}^{\infty}(1+\lambda \mathrm{n})^{\mathrm{m}}\left|\mathrm{a}_{\mathrm{n}}\right|\left|\frac{\mathrm{a}(\mathrm{a}+1)(\mathrm{a}+2)_{\mathrm{n}}-2 \mathrm{a}^{2}(\mathrm{a}+1)_{\mathrm{n}}+\mathrm{a}(\mathrm{a}-1)(\mathrm{a})_{\mathrm{n}} \mid}{(\mathrm{b})_{\mathrm{n}}}\right|}{1-\sum_{\mathrm{n}=1}^{\infty}(1+\lambda \mathrm{n})^{\mathrm{m}}\left|\mathrm{a}_{\mathrm{n}}\right| \frac{\mathrm{a}(\mathrm{a}+1)_{\mathrm{n}}-(\mathrm{a}-1)(\mathrm{a})_{\mathrm{n}}}{(\mathrm{b})_{\mathrm{n}}} \mid}
\end{aligned}
$$

The last expression is bounded by $(1-\alpha)$ if the following inequality which is equivalent to (8) holds:

$$
\sum_{n=1}^{\infty} Y_{n}\left|a_{n}\right|\left|\begin{array}{l}
a(a+1)(a+2)_{n}-a(2 a+\alpha-1) \\
(b+1)_{n}+(a-1)(a+\alpha-1)(a)_{n}
\end{array}\right| \leq 1-\alpha
$$

where $Y_{n}=(1+\lambda n)^{m}$ and this implies $f \in C(m, \lambda, a, b, \alpha)$. This completes the proof of Theorem 2 .

Let $\mathrm{C}^{*}(\mathrm{~m}, \lambda, \mathrm{a}, \mathrm{b}, \alpha)$ denote the class of functions $\mathrm{f} \in \mathcal{A}$ whose Taylor-Maclaurin coefficients $\mathrm{a}_{\mathrm{n}}$ satisfy the condition (8) and denote $\mathrm{C}^{*}[\mathrm{~m}, \lambda, \mathrm{a}, \mathrm{b}, \alpha]=\mathrm{C}^{*}(\mathrm{~m}, \lambda, \mathrm{a}, \mathrm{b}, \alpha) \cap \mathcal{T}$. We note that $\mathrm{C}^{*}(\mathrm{~m}, \lambda, \mathrm{a}, \mathrm{b}, \alpha) \subseteq \mathrm{C}(\mathrm{m}, \lambda, \mathrm{a}, \mathrm{b}, \alpha)$

Corollary 2: Let $\mathrm{f} \in \mathcal{A}$ be in the class $\mathrm{C}^{*}(\mathrm{~m}, \lambda, \mathrm{a}, \mathrm{b}, \alpha)$. Then:

$$
\left|a_{n}\right| \leq \frac{1-\alpha}{(n+1)(n+1-\alpha)(1+\lambda n)^{m}}\left|\frac{(b)_{n}}{(a)_{n}}\right|
$$

Example 3: The function $\mathrm{f}$ given by:

$$
f(z)=z \pm \sum_{n=1}^{\infty} \frac{(1-\alpha) \alpha^{n} \varepsilon_{n}(b)_{n}}{(n+1)(n+1-\alpha)(1+\lambda n)^{m}(a)_{n}} z^{n+1}
$$

belongs to the class $\mathrm{C}^{*}(\mathrm{~m}, \lambda, \mathrm{a}, \mathrm{b}, \alpha)$ for $0 \leq \alpha \leq \frac{1}{2}$, $\mathrm{m} \in \mathbb{Z}, \lambda \geq 0$ and $\varepsilon_{\mathrm{n}} \in \mathbb{C}$ with $\left|\varepsilon_{\mathrm{n}}\right|=1$.

Example 4: For $0 \leq \alpha<1, \lambda \geq 0$ and $m \in \mathbb{N}_{0}$, the following functions defined by:

$$
\begin{aligned}
f_{n}(z)= & z \pm \frac{(1-\alpha)}{(n+1)(n+1-\alpha)(1+\lambda n)^{m}}\left|\frac{(b)_{n}}{(a)_{n}}\right| z^{n+1} \\
& (n=1,2, \ldots ; z \in \mathbb{U})
\end{aligned}
$$

are in the class $C^{*}(m, \lambda, a, b, \alpha)$.
We next study some inclusion properties of the classes $S^{*}(m, \lambda, a, b, \alpha)$ and $C^{*}(m, \lambda, a, b, \alpha)$.

Theorem 3: Let $0 \leq \alpha_{1}<\alpha_{2}<1, \quad 0 \leq \lambda_{1}<\lambda_{2}<1$ and $\mathrm{m}_{1}, \mathrm{~m}_{2} \in \mathbb{Z}$ with $\mathrm{m}_{1}<\mathrm{m}_{2}$. Then:

$\mathrm{S}^{*}\left(\mathrm{~m}, \lambda, \mathrm{a}, \mathrm{b}, \alpha_{2}\right) \quad \subset \mathrm{S}^{*}\left(\mathrm{~m}, \lambda, \mathrm{a}, \mathrm{b}, \alpha_{1}\right)$

$\mathrm{S}^{*}\left(\mathrm{~m}, \lambda_{2}, \mathrm{a}, \mathrm{b}, \alpha\right) \quad \subset \mathrm{S}^{*}\left(\mathrm{~m}, \lambda_{1}, \mathrm{a}, \mathrm{b}, \alpha\right)$

$\mathrm{S}^{*}\left(\mathrm{~m}_{2}, \lambda, \mathrm{a}, \mathrm{b}, \alpha\right) \quad \subset \mathrm{S}^{*}\left(\mathrm{~m}_{1}, \lambda, \mathrm{a}, \mathrm{b}, \alpha\right)$

$\mathrm{S}^{*}(\mathrm{~m}, \lambda, \mathrm{a}+1, \mathrm{~b}, \alpha) \subset \mathrm{S}^{*}(\mathrm{~m}, \lambda, \mathrm{a}, \mathrm{b}, \alpha)$

$\mathrm{S}^{*}(\mathrm{~m}, \lambda, \mathrm{a}, \mathrm{b}+1, \alpha) \supset \mathrm{S}^{*}(\mathrm{~m}, \lambda, \mathrm{a}, \mathrm{b}, \alpha)$

Proof: By using Theorem 1 and Lemma 1.

Theorem 4: Let $0 \leq \alpha_{1}<\alpha_{2}<1, \quad 0 \leq \lambda_{1}<\lambda_{2}<1$ and $\mathrm{m}_{1}, \mathrm{~m}_{2} \in \mathbb{Z}$ with $\mathrm{m}_{1}<\mathrm{m}_{2}$. Then:

$\mathrm{C}^{*}\left(\mathrm{~m}, \lambda, \mathrm{a}, \mathrm{b}, \alpha_{2}\right) \quad \subset \mathrm{C}^{*}\left(\mathrm{~m}, \lambda, \mathrm{a}, \mathrm{b}, \alpha_{1}\right)$

$\mathrm{C}^{*}\left(\mathrm{~m}, \lambda_{2}, \mathrm{a}, \mathrm{b}, \alpha\right) \quad \subset \mathrm{C}^{*}\left(\mathrm{~m}, \lambda_{1}, \mathrm{a}, \mathrm{b}, \alpha\right)$

$\mathrm{C}^{*}\left(\mathrm{~m}_{2}, \lambda, \mathrm{a}, \mathrm{b}, \alpha\right) \quad \subset \mathrm{C}^{*}\left(\mathrm{~m}_{1}, \lambda, \mathrm{a}, \mathrm{b}, \alpha\right)$

$\mathrm{C}^{*}(\mathrm{~m}, \lambda, \mathrm{a}+1, \mathrm{~b}, \alpha) \subset \mathrm{C}^{*}(\mathrm{~m}, \lambda, \mathrm{a}, \mathrm{b}, \alpha)$

$\mathrm{C}^{*}(\mathrm{~m}, \lambda, \mathrm{a}, \mathrm{b}+1, \alpha) \supset \mathrm{C}^{*}(\mathrm{~m}, \lambda, \mathrm{a}, \mathrm{b}, \alpha)$

Proof: By using Theorem 2 and Lemma 1.

Growth and closure theorems: we prove growth theorems when $f \in \mathrm{S}^{*}(\mathrm{~m}, \lambda, \mathrm{a}, \mathrm{b}, \alpha)$ and when $f \in C^{*}(m, \lambda, a, b, \alpha)$. Furthermore, we prove closure theorems when $f$ is in the subclass $S^{*}[m, \lambda, a, b, \alpha]$ and when it is in the subclass $C^{*}[\mathrm{~m}, \lambda, \mathrm{a}, \mathrm{b}, \alpha]$ respectively. 
Theorem 5: Let $f \in \mathrm{S}^{*}(\mathrm{~m}, \lambda, \mathrm{a}, \mathrm{b}, \alpha)$. Then:

$|f(z)| \geq|z|-\frac{1-\alpha}{(2-\alpha)(1+\lambda)^{m}}\left|\frac{b}{a}\right||z|^{2}$

$$
|f(z)| \leq|z|+\frac{1-\alpha}{(2-\alpha)(1+\lambda)^{m}}\left|\frac{b}{a}\right||z|^{2}
$$

for $\mathrm{a}, \mathrm{b} \notin\{0,-1,-2, \ldots\}$ with $|\mathrm{a}+\mathrm{n}| \geq|\mathrm{b}+\mathrm{n}|$ for all $\mathrm{n} \in \mathbb{N}$, $\mathrm{m} \in \mathbb{N}_{0}, \lambda \geq 0,0 \leq \alpha<1$ and $z \in \mathbb{U}$.

Proof: If $\mathrm{f} \in \mathrm{S}^{*}(\mathrm{~m}, \lambda, \mathrm{a}, \mathrm{b}, \alpha)$ with $\mathrm{a}, \mathrm{b} \notin\{0,-1,-2, \ldots\}$, $|\mathrm{a}+\mathrm{n}| \geq|\mathrm{b}+\mathrm{n}|$ for all $\mathrm{n} \in \mathbb{N}, \quad \lambda \geq 0, \quad 0<\alpha<1$ and $\mathrm{m} \in \mathbb{N} \cup\{0\}$, then in view of Theorem 1, we have:

$$
\begin{aligned}
& (2-\alpha)(1+\lambda)^{m}\left|\frac{a}{b}\right| \sum_{n=1}^{\infty}\left|a_{n}\right| \leq \sum_{n=1}^{\infty}(1+\lambda n)^{m} \\
& \left|\frac{a(a+1)_{n}-(a+\alpha-1)(a)_{n}}{(b)_{n}}\right| a_{n} \leq 1-\alpha
\end{aligned}
$$

This yields:

$$
\sum_{n=1}^{\infty}\left|a_{n}\right| \leq \frac{1-\alpha}{(2-\alpha)(1+\lambda)^{m}}\left|\frac{b}{a}\right|
$$

Now:

$$
\begin{aligned}
|f(z)| & \geq|z|-|z|^{2} \sum_{n=1}^{\infty}\left|a_{n}\right| \\
& \geq|z|-\left.\frac{1-\alpha}{(2-\alpha)(1+\lambda)^{m}}\left|\frac{b}{a}\right| z\right|^{2}
\end{aligned}
$$

Also:

$$
|f(z)| \leq|z|+\frac{1-\alpha}{(2-\alpha)(1+\lambda)^{m}}\left|\frac{b}{a}\right||z|^{2}
$$

This completes the proof of Theorem 5 .

Corollary 3: Under the hypothesis of Theorem 5. f(z) is included in a disc centered at the origin with radius $r$ given by:

$$
\mathrm{r}=1+\frac{1-\alpha}{(2-\alpha)(1+\lambda)^{\mathrm{m}}}\left|\frac{\mathbf{b}}{\mathrm{a}}\right|
$$

By using the same proof technique of Theorem 5, we can prove the following result.
Theorem 6: Let $f \in C^{*}(m, \lambda, a, b, \alpha)$. Then:

$$
\begin{aligned}
& |f(z)| \geq|z|-\frac{1-\alpha}{2(2-\alpha)(1+\lambda)^{m}}\left|\frac{b}{a}\right||z|^{2} \\
& |f(z)| \leq|z|+\left.\frac{1-\alpha}{2(2-\alpha)(1+\lambda)^{m}}\left|\frac{b}{a}\right| z\right|^{2}
\end{aligned}
$$

for $\mathrm{a}, \mathrm{b} \notin\{0,-1,-2, \ldots\}$ with $|\mathrm{a}+\mathrm{n}| \geq|\mathrm{b}+\mathrm{n}|$ for all $\mathrm{n} \in \mathbb{N}$, $\mathrm{m} \in \mathbb{N}_{0}, \lambda \geq 0,0 \leq \alpha<1$ and $\mathrm{z} \in \mathbb{U}$.

Corollary 4: Under the hypothesis of Theorem 6. f(z) is included in a disc centered at the origin with radius $r$ given by:

$$
\mathrm{r}=1+\frac{1-\alpha}{2(2-\alpha)(1+\lambda)^{\mathrm{m}}}\left|\frac{\mathrm{b}}{\mathrm{a}}\right|
$$

Corresponding to the subclasses $S^{*}[m, \lambda, a, b, \alpha]$ and $\mathrm{C}^{*}[\mathrm{~m}, \lambda, \mathrm{a}, \mathrm{b}, \alpha]$, we introduce the following closure theorems.

Theorem 7: Let $\mathrm{f}_{0}(\mathrm{z})=\mathrm{z}$ and:

$$
f_{n}(z)=z-\frac{1-\alpha}{(n+1-\alpha)(1+\lambda n)^{m}}\left|\frac{(b)_{n}}{(a)_{n}}\right| z^{n+1}, \quad(n=1,2, \ldots) .
$$

Then $f$ is in the class $S^{*}[m, \lambda, a, b, \alpha]$ if and only if it can be written as:

$$
f(z)=\sum_{n=0}^{\infty} \mu_{n} f_{n}(z)
$$

where $\mu_{n} \geq 0 \quad(\mathrm{n} \geq 0)$ and $\sum_{\mathrm{n}=0}^{\infty} \mu_{\mathrm{n}}=1$.

Proof: Assume that $f(z)=\sum_{n=0}^{\infty} \mu_{n} f_{n}(z)$. Then:

$$
\begin{aligned}
\mathrm{f}(\mathrm{z}) & =\mu_{0} \mathrm{f}_{0}(\mathrm{z})+\sum_{\mathrm{n}=1}^{\infty} \mu_{\mathrm{n}} \mathrm{f}_{\mathrm{n}}(\mathrm{z}) \\
& =\mu_{0} \mathrm{z}+\sum_{\mathrm{n}=1}^{\infty} \mu_{\mathrm{n}}\left[\mathrm{z}-\frac{1-\alpha}{(\mathrm{n}+1-\alpha)(1+\lambda \mathrm{n})^{\mathrm{m}}}\left|\frac{(\mathrm{b})_{\mathrm{n}}}{(\mathrm{a})_{\mathrm{n}}}\right| \mathrm{z}^{\mathrm{n}+1}\right] \\
& =\mu_{0} \mathrm{z}+\sum_{\mathrm{n}=1}^{\infty} \mu_{\mathrm{n}} \mathrm{z}-\sum_{\mathrm{n}=1}^{\infty} \mu_{\mathrm{n}} \frac{1-\alpha}{(\mathrm{n}+1-\alpha)(1+\lambda \mathrm{n})^{\mathrm{m}}}\left|\frac{(\mathrm{b})_{\mathrm{n}}}{(\mathrm{a})_{\mathrm{n}}}\right| \mathrm{z}^{\mathrm{n}+1} \\
& =\mathrm{z}-\sum_{\mathrm{n}=1}^{\infty} \mu_{\mathrm{n}} \frac{1-\alpha}{(\mathrm{n}+1-\alpha)(1+\lambda \mathrm{n})^{\mathrm{m}}}\left|\frac{(\mathrm{b})_{\mathrm{n}}}{(\mathrm{a})_{\mathrm{n}}}\right| \mathrm{z}^{\mathrm{n}+1}
\end{aligned}
$$


Thus:

$$
\begin{aligned}
& \sum_{n=1}^{\infty} \mu_{n}\left(\frac{1-\alpha}{(n+1-\alpha)(1+\lambda n)^{m}}\left|\frac{(b)_{n}}{(a)_{n}}\right|\right) \\
&\left((n+1-\alpha)(1+\lambda n)^{m}\left|\frac{(a)_{n}}{(b)_{n}}\right|\right)=(1-\alpha) \sum_{n=1}^{\infty} \mu_{n} \\
&=(1-\alpha)\left(1-\mu_{0}\right) \leq 1-\alpha
\end{aligned}
$$

Conversely, suppose $\mathrm{f}$ is in the class $\mathrm{S}^{*}[\mathrm{~m}, \lambda, \mathrm{a}, \mathrm{b}, \alpha]$. Since:

$$
\mathrm{a}_{\mathrm{n}} \leq \frac{1-\alpha}{(\mathrm{n}+1-\alpha)(1+\lambda \mathrm{n})^{\mathrm{m}}}\left|\frac{(\mathrm{b})_{\mathrm{n}}}{(\mathrm{a})_{\mathrm{n}}}\right|, \quad(\mathrm{n} \geq 1)
$$

We may set:

$$
\mu_{\mathrm{n}}=\frac{(\mathrm{n}+1-\alpha)(1+\lambda \mathrm{n})^{\mathrm{m}}}{1-\alpha}\left|\frac{(\mathrm{a})_{\mathrm{n}}}{(\mathrm{b})_{\mathrm{n}}}\right| \mathrm{a}_{\mathrm{n}} \quad(\mathrm{n} \geq 1)
$$

and

$$
\mu_{0}=1-\sum_{n=1}^{\infty} \mu_{n}
$$

Then :

$$
\begin{aligned}
f(z) & =z-\sum_{n=1}^{\infty} a_{n} z^{n+1} \\
& =z-\sum_{n=1}^{\infty} \mu_{n} \frac{1-\alpha}{(n+1-\alpha)(1+\lambda n)^{m}}\left|\frac{(b)_{n}}{(a)_{n}}\right| z^{n+1} \\
& =z-\sum_{n=1}^{\infty} \mu_{n}\left(z-f_{n}(z)\right) \\
& =\left(1-\sum_{n=1}^{\infty} \mu_{n}\right) z+\sum_{n=1}^{\infty} \mu_{n} f_{n}(z) \\
& =\sum_{n=0}^{\infty} \mu_{n} f_{n}(z)
\end{aligned}
$$

This completes the proof of Theorem 7.

Corollary 5: The extreme points of the class $\mathrm{S}^{*}[\mathrm{~m}, \lambda, \mathrm{a}, \mathrm{b}, \alpha]$ are the functions $\mathrm{f}_{0}(\mathrm{z})=\mathrm{z}$ and:

$$
f_{n}(z)=z-\frac{1-\alpha}{(n+1-\alpha)(1+\lambda n)^{m}}\left|\frac{(b)_{n}}{(a)_{n}}\right| z^{n+1}, \quad(n=1,2, \ldots) .
$$

By using the same proof technique of Theorem 7, we can prove the following theorem.

Theorem 8: Let $\mathrm{f}_{0}(\mathrm{z})=\mathrm{z}$ and:

$$
f_{n}(z)=z-\frac{1-\alpha}{(n+1)(n+1-\alpha)(1+\lambda n)^{m}}\left|\frac{(b)_{n}}{(a)_{n}}\right| z^{n+1},(n=1,2, \ldots)
$$

Then $\mathrm{f}$ is in the class $\mathrm{C}^{*}[\mathrm{~m}, \lambda, \mathrm{a}, \mathrm{b}, \alpha]$ if and only if it can be written as:

$$
f(z)=\sum_{n=0}^{\infty} \mu_{n} f_{n}(z)
$$

where, $\mu_{n} \geq 0 \quad(n \geq 0)$ and $\sum_{n=0}^{\infty} \mu_{n}=1$.

Corollary 6: The extreme points of the class $\mathrm{C}^{*}[\mathrm{~m}, \lambda, \mathrm{a}, \mathrm{b}, \alpha]$ are the functions $\mathrm{f}_{0}(\mathrm{z})=\mathrm{z}$ and:

$$
f_{n}(z)=z-\frac{1-\alpha}{(n+1)(n+1-\alpha)(1+\lambda n)^{m}}\left|\frac{(b)_{n}}{(a)_{n}}\right| z^{n+1},(n=1,2, \ldots)
$$

Subordination results: we obtain a sharp subordination results associated with the classes $\mathrm{S}^{*}(\mathrm{~m}, \lambda, \mathrm{a}, \mathrm{b}, \alpha)$ and $\mathrm{C}^{*}(\mathrm{~m}, \lambda, \mathrm{a}, \mathrm{b}, \alpha)$ by using the same technique as $i^{[12-15]}$. Some applications of those main results which give important results of analytic functions are also investigated.

Theorem 9: Let $f \in \mathrm{S}^{*}(\mathrm{~m}, \lambda, \mathrm{a}, \mathrm{b}, \alpha) \quad$ where $\mathrm{a}, \mathrm{b} \notin\{0,-1,-2, \ldots\}$ with $|\mathrm{a}+\mathrm{n}| \geq|\mathrm{b}+\mathrm{n}|$ for all $\mathrm{n} \in \mathbb{N}$, $\mathrm{m} \in \mathbb{N}_{0}, \lambda \geq 0$ and $0 \leq \alpha<1$. Then:

$$
\frac{(2-\alpha)(1+\lambda)^{\mathrm{m}}\left|\frac{\mathrm{a}}{\mathrm{b}}\right|}{2(1-\alpha)+2(2-\alpha)(1+\lambda)^{\mathrm{m}}\left|\frac{\mathrm{a}}{\mathrm{b}}\right|}(\mathrm{f} * \mathrm{~g})(\mathrm{z}) \prec \mathrm{g}(\mathrm{z}) \quad(\mathrm{z} \in \mathbb{U})
$$

for every $\mathrm{g}$ in $\mathrm{K}$ and:

$$
\Re \mathrm{f}(\mathrm{z})>-\left(1+\frac{1-\alpha}{(2-\alpha)(1+\lambda)^{\mathrm{m}}}\left|\frac{\mathrm{b}}{\mathrm{a}}\right|\right)
$$

The constant $1+\left((1-\alpha) /(2-\alpha)(1+\lambda)^{\mathrm{m}}\right)\left|\frac{\mathrm{b}}{\mathrm{a}}\right|$ cannot be replaced by a larger one.

Proof: Let $f \in \mathrm{S}^{*}(\mathrm{~m}, \lambda, \mathrm{a}, \mathrm{b}, \alpha)$ and let:

$$
\mathrm{g}(\mathrm{z})=\mathrm{z}+\sum_{\mathrm{n}=1}^{\infty} \mathrm{c}_{\mathrm{n}} \mathrm{z}^{\mathrm{n}+1}
$$


be any function in the class $\mathrm{K}$. Then we readily have:

$$
\begin{aligned}
& \frac{(2-\alpha)(1+\lambda)^{\mathrm{m}}\left|\frac{\mathrm{a}}{\mathrm{b}}\right|}{2(1-\alpha)+2(2-\alpha)(1+\lambda)^{\mathrm{m}}\left|\frac{\mathrm{a}}{\mathrm{b}}\right|}(\mathrm{f} * \mathrm{~g})(\mathrm{z}) \\
& =\frac{(2-\alpha)(1+\lambda)^{\mathrm{m}}\left|\frac{\mathrm{a}}{\mathrm{b}}\right|}{2(1-\alpha)+2(2-\alpha)(1+\lambda)^{\mathrm{m}}\left|\frac{\mathrm{a}}{\mathrm{b}}\right|}\left(\mathrm{z}+\sum_{\mathrm{n}=1}^{\infty} \mathrm{a}_{\mathrm{n}} \mathrm{c}_{\mathrm{n}} \mathrm{z}^{\mathrm{n}+1}\right)
\end{aligned}
$$

Thus, the subordination result (9) will hold true if the sequence:

$$
\begin{aligned}
& \left\{\frac{(2-\alpha)(1+\lambda)^{m}\left|\frac{a}{b}\right| a_{n}}{2(1-\alpha)+2(2-\alpha)(1+\lambda)^{m}\left|\frac{a}{b}\right|}\right\}_{n=1}^{\infty} \\
& \Re\left\{1+\sum_{\mathrm{n}=0}^{\infty} \frac{(2-\alpha)(1+\lambda)^{\mathrm{m}}\left|\frac{\mathrm{a}}{\mathrm{b}}\right|}{1-\alpha+(2-\alpha)(1+\lambda)^{\mathrm{m}}\left|\frac{\mathrm{a}}{\mathrm{b}}\right|^{\mathrm{n}} \mathrm{a}_{\mathrm{n}} \mathrm{z}^{\mathrm{n}+1}}\right\}=\Re\left\{\begin{array}{l}
1+\frac{(2-\alpha)(1+\lambda)^{\mathrm{m}}\left|\frac{\mathrm{a}}{\mathrm{b}}\right|}{1-\alpha+(2-\alpha)(1+\lambda)^{\mathrm{m}}\left|\frac{\mathrm{a}}{\mathrm{b}}\right|^{\mathrm{z}}} \\
+\frac{(2-\alpha)}{1-\alpha+(2-\alpha)(1+\lambda)^{\mathrm{m}}\left|\frac{\mathrm{a}}{\mathrm{b}}\right|} \sum_{\mathrm{n}=1}^{\infty}(1+\lambda)^{\mathrm{m}}\left|\frac{\mathrm{a}}{\mathrm{b}}\right| \mathrm{a}_{\mathrm{n}} \mathrm{z}^{\mathrm{n}+1}
\end{array}\right\} \\
& >1-\frac{(2-\alpha)(1+\lambda)^{\mathrm{m}}\left|\frac{\mathrm{a}}{\mathrm{b}}\right|}{1-\alpha+(2-\alpha)(1+\lambda)^{\mathrm{m}}\left|\frac{\mathrm{a}}{\mathrm{b}}\right|^{\mathrm{r}}} \\
& -\frac{1}{1-\alpha+(2-\alpha)(1+\lambda)^{m}\left|\frac{a}{b}\right|} \sum_{n=1}^{\infty}(n+1-\alpha)(1+\lambda n)^{m}\left|\frac{(a)_{n}}{(b)_{n}}\right| a_{n} r^{n+1} \\
& \geq 1-\frac{(2-\alpha)(1+\lambda)^{\mathrm{m}}\left|\frac{\mathrm{a}}{\mathrm{b}}\right|}{1-\alpha+(2-\alpha)(1+\lambda)^{\mathrm{m}}\left|\frac{\mathrm{a}}{\mathrm{b}}\right|} \mathrm{r}-\frac{1-\alpha}{1-\alpha+(2-\alpha)(1+\lambda)^{\mathrm{m}}\left|\frac{\mathrm{a}}{\mathrm{b}}\right|} \mathrm{r}>0 \quad(|\mathrm{z}|=\mathrm{r}) \text {. }
\end{aligned}
$$$$
(\mathrm{n}+1-\alpha)(1+\lambda \mathrm{n})^{\mathrm{m}}\left|\frac{(\mathrm{a})_{\mathrm{n}}}{(\mathrm{b})_{\mathrm{n}}}\right|
$$$$
\text { is subordinating factor sequence, with } \mathrm{a}_{0}=1 \text {. In view of }
$$$$
\text { Lemma 2, this is equivalent to the following inequality: }
$$

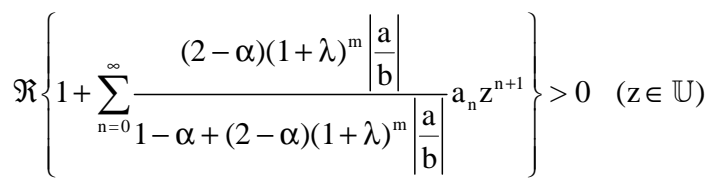$$
\text { Now, since: }
$$

This proves the inequality (11) and hence also the subordination result (9) asserted by Theorem 9. The inequality (10) follows from (9) by taking:

$$
\mathrm{g}(\mathrm{z})=\frac{\mathrm{z}}{1-\mathrm{z}} \in \mathrm{K}
$$

Next, we consider the function:

$$
f_{1}(z)=z-\frac{(1-\alpha)}{(2-\alpha)(1+\lambda)^{m}}\left|\frac{b}{a}\right| z^{2}
$$

which satisfies the assumption of Theorem 9. Then by using (9), we have: 


$$
\frac{(2-\alpha)(1+\lambda)^{\mathrm{m}}\left|\frac{\mathrm{a}}{\mathrm{b}}\right|}{2(1-\alpha)+2(2-\alpha)(1+\lambda)^{\mathrm{m}}\left|\frac{\mathrm{a}}{\mathrm{b}}\right|} \mathrm{f}_{1}(\mathrm{z}) \prec \frac{\mathrm{z}}{1-\mathrm{z}} \quad(\mathrm{z} \in \mathbb{U})
$$

It can be easily verified for the function $\mathrm{f}_{1}(\mathrm{z})$ that:

$$
\inf _{z \in \mathbb{U}}\left\{\Re\left(\frac{(2-\alpha)(1+\lambda)^{\mathrm{m}}\left|\frac{\mathrm{a}}{\mathrm{b}}\right|}{2(1-\alpha)+2(2-\alpha)(1+\lambda)^{\mathrm{m}}\left|\frac{\mathrm{a}}{\mathrm{b}}\right|} \mathrm{f}_{1}(\mathrm{z})\right)\right\}=-\frac{1}{2} \quad(\mathrm{z} \in \mathbb{U})
$$

which completes the proof of Theorem 9 .

By taking $\lambda=0$ in Theorem 9 , we have the following corollary.

Corollary 7: If the function $f$ defined by (1) satisfies:

$$
\sum_{n=1}^{\infty}(n+1-\alpha)\left|\frac{(a)_{n}}{(b)_{n}}\right|\left|a_{n}\right| \leq 1-\alpha
$$

where $a, b \notin\{0,-1,-2, \ldots\}$ with $|a+n| \geq|b+n|$ for all $\mathrm{n} \in \mathbb{N}$ and $0 \leq \alpha<1$, then for every function $\mathrm{g}$ in $\mathrm{K}$, one has:

$$
\frac{2-\alpha}{2(1-\alpha)\left|\frac{\mathrm{b}}{\mathrm{a}}\right|+2(2-\alpha)}(\mathrm{f} * \mathrm{~g})(\mathrm{z}) \prec \mathrm{g}(\mathrm{z}) \quad(\mathrm{z} \in \mathbb{U})
$$

and

$$
\Re \mathrm{f}(\mathrm{z})>-\left(1+\frac{1-\alpha}{2-\alpha}\left|\frac{\mathrm{b}}{\mathrm{a}}\right|\right)
$$

The constant $1+(1-\alpha)\left|\frac{\mathbf{b}}{\mathrm{a}}\right| /(2-\alpha) \quad$ cannot be replaced by a larger one.

Putting $\lambda=0$ and $\mathrm{a}=\mathrm{b}$ in Theorem 9, we have the following corollary.

Corollary 8: If the function $f$ defined by (1) satisfies:

$$
\sum_{n=1}^{\infty}(n+1-\alpha)\left|a_{n}\right| \leq 1-\alpha, \quad(0 \leq \alpha<1)
$$

then for every function $\mathrm{g}$ in $\mathrm{K}$, one has:

$$
\frac{2-\alpha}{2(3-2 \alpha)}(f * g)(z) \prec g(z) \quad(z \in \mathbb{U})
$$

and

$$
\Re \mathrm{f}(\mathrm{z})>-\left(\frac{3-2 \alpha}{2-\alpha}\right)
$$

The constant $(3-2 \alpha) /(2-\alpha)$ cannot be replaced by a larger one.

Letting $\lambda=0, a=2$ and $b=2-\alpha ; 0 \leq \alpha<1$ in Theorem 9 , we have the following corollary related to the starlikeness of the fractional derivative and fractional integral operator $\Omega^{\alpha} \mathrm{f}(\mathrm{z})$ due to Owa and Srivastava which is mentioned above.

Corollary 9: If the function $\mathrm{f}$ defined by (1) satisfies:

$$
\sum_{n=1}^{\infty}(n+1-\alpha) \frac{(n+1) !}{(2-\alpha)_{n}}\left|a_{n}\right| \leq 1-\alpha(0<\alpha<1)
$$

then for every function $\mathrm{g}$ in $\mathrm{K}$, one has:

$$
\frac{1}{3-\alpha}(\mathrm{f} * \mathrm{~g})(\mathrm{z}) \prec \mathrm{g}(\mathrm{z}) \quad(\mathrm{z} \in \mathbb{U})
$$

and

$$
\Re \mathrm{f}(\mathrm{z})>-\left(\frac{3-\alpha}{2}\right)
$$

The constant $(3-\alpha) / 2$ cannot be replaced by a larger one.

It is clear from the proof of Theorem 9 that the function $\mathrm{f}(\mathrm{z})=\mathrm{z}-\frac{1-\alpha}{2-\alpha} \mathrm{z}^{2} \quad(0 \leq \alpha<1 ; \mathrm{z} \in \mathbb{U}) \quad$ is an extremal function of Corollary 8. Also the following example gives a non-polynomial function satisfies the same corollary.

Example 5: Let the function h be defined by:

$$
\mathrm{h}(\mathrm{z})=\frac{\mathrm{z}}{1+\frac{1-\alpha}{2(3-2 \alpha)} \mathrm{z}} \quad(0 \leq \alpha<1 ; \mathrm{z} \in \mathbb{U})
$$

the above function is analytic in $\mathbb{U}$ and it is equivalent to:

$$
h(z)=z+\sum_{n=1}^{\infty}\left(\frac{\alpha-1}{2(3-2 \alpha)}\right)^{n} z^{n+1}
$$

Then we have: 


$$
\sum_{n=1}^{\infty}(n+1-\alpha)\left|\frac{\alpha-1}{2(3-2 \alpha)}\right|^{n} \leq \sum_{n=1}^{\infty} 2^{n}\left|\frac{\alpha-1}{2(3-2 \alpha)}\right|^{n}=\frac{1-\alpha}{4-3 \alpha} \leq 1-\alpha
$$

Therefore, the Taylor-Maclaurin coefficients of the function h satisfy the condition in Corollary 8 .

By the same proof technique of Theorem 9, we can prove the following theorem.

Theorem 10: Let $f \in C^{*}(m, \lambda, a, b, \alpha) \quad$ where $a, b \notin\{0,-1,-2, \ldots\}$ with $|a+n| \geq|b+n|$ for all $n \in \mathbb{N}$, $\mathrm{m} \in \mathbb{N}_{0}, \lambda \geq 0$ and $0 \leq \alpha<1$. Then:

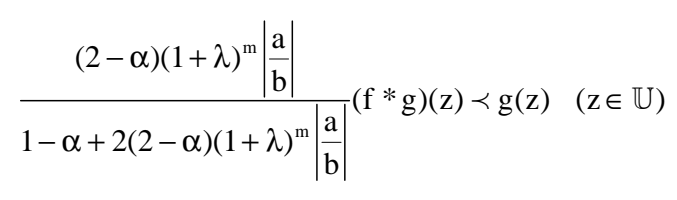

for every $\mathrm{g}$ in $\mathrm{K}$ and:

$$
\Re \mathrm{f}(\mathrm{z})>-\left(1+\frac{1-\alpha}{2(2-\alpha)(1+\lambda)^{\mathrm{m}}}\left|\frac{\mathrm{b}}{\mathrm{a}}\right|\right)
$$

The constant $1+\left((1-\alpha) / 2(2-\alpha)(1+\lambda)^{\mathrm{m}}\right)\left|\frac{\mathrm{b}}{\mathrm{a}}\right|$ cannot be replaced by a larger one.

By taking $\lambda=0$ in Theorem 10, we have the following corollary.

Corollary 10: If the function $\mathrm{f}$ defined by (1) satisfies:

$$
\sum_{n=1}^{\infty}(n+1)(n+1-\alpha)\left|\frac{(a)_{n}}{(b)_{n}}\right| a_{n} \mid \leq 1-\alpha
$$

where $a, b \notin\{0,-1,-2, \ldots\}$ with $|a+n| \geq|b+n|$ for all $\mathrm{n} \in \mathbb{N}$ and $0 \leq \alpha<1$, then for every function $\mathrm{g}$ in $\mathrm{K}$, one has:

$$
\frac{2-\alpha}{2(2-\alpha)+(1-\alpha)\left|\frac{\mathrm{b}}{\mathrm{a}}\right|}(\mathrm{f} * \mathrm{~g})(\mathrm{z}) \prec \mathrm{g}(\mathrm{z}) \quad(\mathrm{z} \in \mathbb{U})
$$

and

$$
\Re \mathrm{f}(\mathrm{z})>-\left(1+\frac{1-\alpha}{2(2-\alpha)}\left|\frac{\mathrm{b}}{\mathrm{a}}\right|\right)
$$

The constant $1+(1-\alpha)\left|\frac{\mathrm{b}}{\mathrm{a}}\right| / 2(2-\alpha)$ cannot be replaced by a larger one.
Putting $\lambda=0$ and $\mathrm{a}=\mathrm{b}$ in Theorem 10, we have the following corollary.

Corollary 11: If the function $f$ defined by (1) satisfies:

$$
\sum_{n=1}^{\infty}(n+1)(n+1-\alpha)\left|a_{n}\right| \leq 1-\alpha \quad(0 \leq \alpha<1)
$$

then for every function $\mathrm{g}$ in $\mathrm{K}$, one has:

$$
\frac{2-\alpha}{5-3 \alpha}(\mathrm{f} * \mathrm{~g})(\mathrm{z}) \prec \mathrm{g}(\mathrm{z}) \quad(\mathrm{z} \in \mathbb{U})
$$

and

$$
\Re \mathrm{f}(\mathrm{z})>-\left(\frac{5-3 \alpha}{4-2 \alpha}\right)
$$

The constant $(5-3 \alpha) /(4-2 \alpha)$ cannot be replaced by a larger one.

Letting $\lambda=0, a=2$ and $b=2-\alpha, 0 \leq \alpha<1$ in Theorem 10 , we have the following corollary related to the convexity of the fractional derivative and fractional integral operator $\Omega^{\alpha} \mathrm{f}(\mathrm{z})$ due to Owa and Srivastava which is mentioned above.

Corollary 12: If the function $f$ defined by (1) satisfies:

$$
\sum_{n=1}^{\infty}(n+1)(n+1-\alpha) \frac{(n+1) !}{(2-\alpha)_{n}}\left|a_{n}\right| \leq 1-\alpha(0 \leq \alpha<1)
$$

then for every function $\mathrm{g}$ in $\mathrm{K}$, one has:

$$
\frac{2}{5-\alpha}(\mathrm{f} * \mathrm{~g})(\mathrm{z}) \prec \mathrm{g}(\mathrm{z}) \quad(\mathrm{z} \in \mathbb{U})
$$

and

$$
\Re \mathrm{f}(\mathrm{z})>-(5-\alpha)
$$

The $5-\alpha$ cannot be replaced by a larger one.

Note that the function $f(z)=z-\frac{1-\alpha}{2(2-\alpha)} z^{2}$ $(0 \leq \alpha<1 ; z \in \mathbb{U})$ is an extremal function of Corollary 11. Also the following example gives a non-polynomial function satisfies the same corollary.

Example 6: Let the function $\mathrm{h}$ be defined by: 


$$
\mathrm{g}(\mathrm{z})=\frac{\mathrm{z}}{1+\frac{1-\alpha}{4(3-\alpha)} \mathrm{z}} \quad(0 \leq \alpha<1 ; \mathrm{z} \in \mathbb{U})
$$

The above function is analytic in $\mathbb{U}$ and it is equivalent to:

$$
g(z)=z+\sum_{n=1}^{\infty}\left(\frac{\alpha-1}{4(3-\alpha)}\right)^{n} z^{n+1}
$$

Then we have:

$$
\sum_{n=1}^{\infty}(n+1)(n+1-\alpha)\left|\frac{\alpha-1}{4(3-\alpha)}\right|^{n} \leq \sum_{n=1}^{\infty} 4^{n}\left|\frac{\alpha-1}{4(3-\alpha)}\right|^{n}=\frac{1-\alpha}{2(2-\alpha)} \leq 1-\alpha
$$

Therefore, the Taylor-Maclaurin coefficients of the function $g$ satisfy the condition in Corollary 11 .

\section{DISCUSSION}

Let $\mathcal{A}$ denote the set of functions $\mathrm{f}$ of the form $\mathrm{f}(\mathrm{z})=\mathrm{z}+\mathrm{a}_{1} \mathrm{z}^{2}+\mathrm{a}_{2} \mathrm{z}^{3}+\ldots$, which are analytic in the open unit disk. A new bijective convolution linear operator defined on $\mathcal{A}$ is introduced, which is a generalization of Carlson-Shaffer operator ${ }^{[2]}$ and various well known operators and classes of analytic functions involving that operator are studied. Mainly, several properties of some subclasses are investigated, like coefficient bounds, inclusions, growth and closure theorems. Furthermore, main subordination results with some applications are investigated as well. The proof thechnique of those subordination results is used earlier by many researchers, namely Srivastava, Attyia, Ali, Ravichandran and Seenivasagan.

\section{CONCLUSION}

We conclude this study with some suggestions for future research, one direction is to study other classes of analytic functions involving our operator $\mathrm{J}(\mathrm{m}, \lambda, \mathrm{a}, \mathrm{b})$. Another direction would be studying other properties of the classes $S(m, \lambda, a, b, \alpha)$ and $C(m, \lambda, a, b, \alpha)$.

\section{ACKNOWLEDGMENT}

The study showed here was supported by EScienceFund 04-01-02-SF0425, MOSTI, Malaysia.

\section{REFERENCES}

1. Ruscheweyh, S., 1977. Linear operator between classes of prestarlike functions. Commentarii Mathematici Helvetici, 52: 497-509. DOI: 10.1007/BF02567382
2. Carlson, B.C. and D.B. Shaffer, 1984. Starlike and prestarlike hypergeometric functions. SIAM. J. Math. Anal., 15: 737-745. http://scitation.aip.org/getabs/servlet/GetabsServlet ?prog=normal\&id=SJMAAH00001500000400073 7000001\&idtype $=$ cvips \&gifs $=$ yes

3. Ruscheweyh, S., 1975. New criteria for univalent functions. Proc. Am. Math. Soc., 49: 109-115. http://www.jstor.org/pss/2039801

4. Al-Oboudi, F.M., 2004. On univalent functions defined by a generalized $\mathrm{S} \breve{a} \mathrm{lă}$ gean operator. Int. J. Math. Math. Sci., 27: 1429-1436. DOI: $10.1155 / \mathrm{S} 0161171204108090$

5. S ă lă gean, G.S., 1983. Subclasses of univalent functions. Lecture Notes Math., 1013: 362-372. DOI: $10.1007 / \mathrm{BFb} 0066543$

6. Owa, S. and H.M. Srivastava, 1987. Univalent and starlike generalized hypergeometric functions. Can. J. Math., 39: 1057-1077.

7. Inayat Noor, k., 1999. On new classes of integral operators. J. Natural Geomet., 16: 71-80.

8. Inayat Noor, k. and M.A. Noor, 1999. On integral operators. J. Math. Anal. Appli, 238: 341-352.

9. Choi, J.H., M. Saigo and H.M. Srivastava, 2002. Some inclusion properties of a certain family of integral operators. J. Math. Anal. Appli, 276: 432-445. DOI: $10.1016 / \mathrm{S} 0022-247 \mathrm{X}(02) 00500-0$

10. Goodman, A.W., 1983. Univalent Functions. Mariner Publishing Company, Tampa, Florida, pp: 84-88, 135.

11. Wilf, H.S., 1961. Subordinating factor sequences for covex maps of the unit circle. Proc. Am. Math. Soc., 12: 689-693.

12. Srivastava, H.M. and A.A. Attiya, 2004. Some subordination results associated with certain subclasses of analytic functions. J. Inequal. Pure Applied Math., 5: 1-6. http://www.emis.de/journals/JIPAM/images/113_0 4_JIPAM/113_04_www.pdf

13. Attiya, A.A., 2005 . On some applications of a subordination theorem. J. Math. Anal. Appli., 311: 489-494. DOI: 10.1016/j.jmaa.2005.02.056

14. Attiya, A.A., Nak Eun Cho and M.A. Kutbi, 2008. Subordination properties for certain analytic functions. Int. J. Math. Math. Sci., 2008: 8-8. DOI: $10.1155 / 2008 / 638251$

15. Ali, R.M., V. Ravichandran, N. Seenivasagan, 2006. Subordination by convex functions. Int. J. Math. Math. Sci., 24: 563-568. DOI: 10.1155/IJMMS/2006/62548 